\title{
Cytokeratin expression in adrenal phaeochromocytomas and extra-adrenal paragangliomas
}

\author{
R Chetty, P Pillay, V Jaichand
}

\begin{abstract}
Aim-To examine whether adrenal phaeochromocytomas and extra-adrenal paragangliomas are immunoreactive for commercially available and routinely used cytokeratin antibodies.

Methods-18 extra-adrenal paragangliomas and seven adrenal phaeochromocytomas were stained with CAM 5.2, AE1/3, and 34/E12 following microwave antigen retrieval of formalin fixed tissue.

Results-A single case from the cauda equina was positive for both CAM 5.2 and AE1/3. In addition, two other cases-an intravagal and an orbital paraganglioma-also showed strong immunopositivity with CAM 5.2 and AE1/3. All phaeochromocytomas were negative with all epithelial markers.

Conclusions-Cauda equina paragangliomas are known to stain with cytokeratins; however, occasional paragangliomas from other sites may also be immunoreactive with cytokeratins. If the results of immunohistochemistry are not interpreted in the clinical and morphological context, the failure to recognise that extra-adrenal paragangliomas may on occasion react with anticytokeratin antibodies may lead to their being confused with metastatic carcinomas.

(f Clin Pathol 1998;51:477-478)
\end{abstract}

Keywords: paraganglioma; phaeochromocytoma; cytokeratin

The paraganglion system can be divided into (1) the adrenal medulla and (2) the extraadrenal paraganglion system. The latter can be further subdivided into two components: first, that associated with the orthosympathetic system (occurring in the para-aortic, thoracic, and abdominal regions and functionally related to the adrenal medulla); and second, that related to the parasympathetic system. ${ }^{1}$

Cytokeratin expression has been used to distinguish between paragangliomas, which are negative, and carcinoids and neuroendocrine carcinomas, which are positive. ${ }^{23}$ However, paragangliomas of the cauda equina have a distinctive cytokeratin immunophenotype. ${ }^{45}$ Paragangliomas from various sites have been examined for a host of neural and neuroendocrine markers, and some of these studies have also looked cytokeratin expression. ${ }^{6-10}$ Thus far, only one study has shown cytokeratin expression in paragangliomas not arising from the cauda equina. ${ }^{10}$ The purpose of this paper is to verify those findings, and also to establish if cytokeratin can be identified in phaeochromocytomas of the adrenal gland and in extraadrenal paragangliomas using commonly available anticytokeratin antibodies.

\section{Methods}

CLINICAL DETAILS

Extra-adrenal paragangliomas

Eighteen cases were studied, including one from the cauda equina. Other sites included the orbit, organ of Zuckerkandl, carotid body, and vagus nerve. The patients ranged in age from seven to 66 years, and included 13 females and five males.

\section{Adrenal phaeochromocytomas}

Seven cases were studied in patients ranging in age from 15 to 74 years.

\section{MATERIALS}

All material was fixed in $10 \%$ buffered formalin, processed in a routine fashion, and embedded in paraffin wax. In addition to routine haematoxylin and eosin stains, immunohistochemistry was performed on the paraffin embedded tissue using the streptavidin-biotin complex technique with DAB as chromogen. All tumours were stained for chromogranin A, neurone specific enolase, and S-100 protein. For the purpose of this study three anticytokeratin antibodies were also used: CAM 5.2 (Biogenix, San Ramon, California, USA; dilution 1 in 400), AE1/3 (Signet Laboratories, Dedham, Massachusetts, USA; dilution 1 in 1600), and 34ßE12 (Dakopatts, High Wycombe, UK; prediluted). Appropriate positive and negative controls were run in parallel. All positive cases were also stained without microwave retrieval.

\section{Results}

All the cases were typical of paragangliomas or phaeochromocytomas, being composed of chief and sustentacular cells. In addition, all cases were positive for chromogranin A and NSE (chief cells) and S-100 protein (sustentacular cells).

The case from the cauda equina showed strong CAM 5.2 positivity, with weaker staining with AE1/3. Only two other cases showed immunoreactivity with CAM 5.2 and AE1/3. One case was an orbital paraganglioma and the other an intravagal paraganglioma. In both these cases staining was diffuse, intense, and involved all the cells (fig 1). 


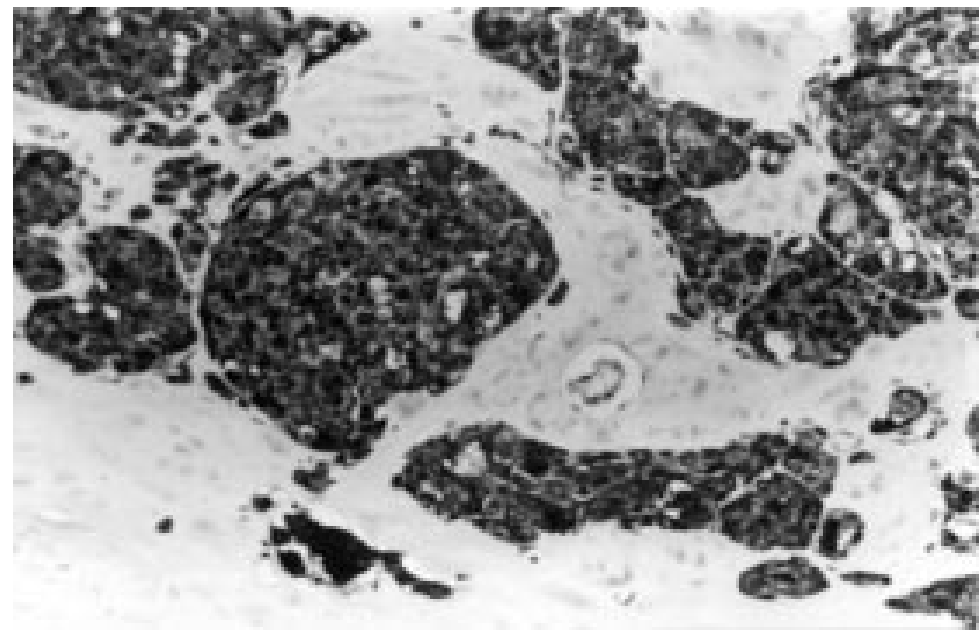

Figure 1 A case of intravagal paraganglioma stained with CAM 5.2. Note there is intense, unequivocal cytoplasmic immunostaining.

The adrenal phaeochromocytomas were negative for both CAM 5.2 and AE1/3. Both adrenal and extra-adrenal cases were negative for $34 \beta \mathrm{E} 12$.

The cases that were positive with microwave antigen retrieval were also positive when stained without antigen retrieval, but the degree of staining was less intense.

\section{Discussion}

Extra-adrenal paragangliomas are unique tumours of the dispersed neuroendocrine system that differ from other tumours of this system in their clinical behaviour. Indeed, the behaviour is site dependent. ${ }^{1}$ Extra-adrenal paragangliomas, unlike their adrenal counterparts, rarely result in clinical manifestations related to excess hormone production. Although the majority of paragangliomas are sporadic, they can also be multicentric and familial - that is, they can be part of the multiple endocrine neoplasia syndromes.

Paragangliomas in the head and neck often raise the suspicion of lymphadenopathy from metastatic carcinoma. From a diagnostic point of view this confusion is heightened by cytokeratin expression in occasional paragangliomas. The absence of cytokeratin expression by paragangliomas has been used to separate them from other neuroendocrine tumours. This finding was confirmed and accepted until Johnson and colleagues noted that some head and neck paragangliomas did show cytokeratin immunoexpression. ${ }^{10}$ However, the extent and diagnostic implications of these findings were not developed.

Ultrastructural examination of paragangliomas has not revealed the presence of tonofila- ments, although cell adhesion specialisations are seen. ${ }^{1}$ These are regarded as modified tight junctions rather than true desmosomes and are the basis for the lack of immunoreactivity with anti-cytokeratins. Paragangliomas of the cauda equina, on the other hand, have been shown to contain cytoplasmic intermediate filaments and thus are immunoreactive for cytokeratins. ${ }^{5}$ Cytokeratin immunoexpression has also been demonstrated in gangliocytic paragangliomas. ${ }^{11}$

Two of 18 paragangliomas not arising from the cauda equina have been found to show intense, diffuse immunoreactivity for both CAM 5.2 and AE1/3. This finding is important from a diagnostic point of view because these lesions may be misinterpreted as carcinomas. However, this can be avoided if paragangliomas are considered in the differential diagnosis and the morphological features are taken into account. Furthermore, one should be aware that very occasional non-cauda-equina paragangliomas express the commonly used antibodies to cytokeratin (CAM 5.2 and AE1/3). Phaeochromocytomas, on the other hand, appear to be negative for both these markers.

The rationale for this immunoexpression is not readily apparent. This study adds another group of tumours that must be considered when assessing cytokeratin positivity in the diagnostic setting. ${ }^{12}$

1 Kliewer KE, Cochran AJ. A review of the histology, ultrastructure, immunohistology, and molecular biology of extra-adrenal paragangliomas. Arch Pathol Lab Med 1989;113:1209-18.

2 Gould VE, Wiedenmann B, Lee I, et al. Synaptophysin expression in neuroendocrine neoplasms as determined by immunocytochemistry. Am f Pathol 1987;126:243-57.

3 Sibley RK. The intermediate filament profile of neuroendocrine neoplasms [abstr]. Lab Invest 1985;52:62A.

4 Ironside JW, Royds JA, Taylor CD, et al. Paraganglioma of the cauda equina: a histological, ultrastructural and immunocytochemical study of two cases with review of the literature. F Pathol 1985;145:195-201.

5 Orrell JM, Hales SA. Paragangliomas of the cauda equina have a distinctive cytokeratin immunophenotype. Histopathology 1992;21:479-81.

6 Googe PB, Ferry JA, Bhan AK, et al. A comparison of paraganglioma, carcinoid tumor, and small-cell carcinoma of the larynx. Arch Pathol Lab Med 1988;112:809-15.

7 Milroy CM, Rode J, Moss E. Laryngeal paragangliomas and neuroendocrine carcinomas. Histopathology 1991;18:2019.

8 Moran CA, Albores-Saavedra J, Wenig BM, et al. Pigmented extraadrenal paraganglioma. Cancer 1997;79:398-402.

9 Moran CA, Suster S, Fishback N, et al. Mediastinal paragangliomas. A clinicopathologic and immunohistochemical study of 16 cases. Cancer 1993;72:2358-64.

10 Johnson TL, Zarbo RJ, Lloyd RV, et al. Paragangliomas of the head and neck: immunohistochemical, neuroendocrine and intermediate filament typing. Mod Pathol 1988;1:21623.

11 Furihata $\mathrm{M}$, Sonobe $\mathrm{H}$, Iwata J, et al. Immunohistochemical characterization of a case of gangliocytic paraganglioma. Pathol Int 1996;46:610-13.

12 Heatley MK. Cytokeratins and cytokeratin staining in diagnostic histopathology. Histopathology 1996;28:479-83. 